\title{
25 Research Soure \\ The Value of Dual-Energy CT Imaging In The Clinical Diagnosis of Gout
}

\section{Xiulin GAO GAO ( $\nabla$ ji20210629@163.com )}

fenyang College of Shanxi Medical University

Huanling GAO

fenyang College of Shanxi Medical University

\section{Liyun ZHANG}

shanxi Norman Bethune Hospital

\section{Liqing ZHANG}

fenyang Hospital of Shanxi Province

\section{Research Article}

Keywords: arthritis, gout, CT, dual-energy, diagnosis

Posted Date: September 21st, 2021

DOl: https://doi.org/10.21203/rs.3.rs-849377/v1

License: (c) (1) This work is licensed under a Creative Commons Attribution 4.0 International License. Read Full License 


\section{Abstract}

Objective To explore the clinical diagnostic value of dual-energy CT imaging technology in patients with gout.

Methods A total of 83 suspected gout patients admitted from June 2019 to December 2020 were selected as research subjects. Dual-energy CT and gold standard examination were performed on all patients. The detection rates of urate crystals were compared between the two methods. Clinicians performed physical examinations on patients with confirmed gout, and the examination results were compared with the results of urate crystals found by dual-energy CT.

Results The sensitivity, specificity, negative predictive value, and positive predictive value of dual-energy CT imaging in diagnosing gout were $92.31 \%, 83.33 \%, 75 \%$, and $95.24 \%$, respectively. The kappa value was 0.73 , and the area under the ROC curve was 0.88 (95\% confidence interval, $0.77 \llbracket 0.99, P<0.01$ ). Compared with clinical physical examinations, the number of urate crystal deposits detected by dualenergy CT imaging was 4.40 times that of the former. The detection rate of urate crystals and bone destruction in patients with chronic intermittent gout by dual-energy CT examination was higher than that in the acute phase. (The $\chi^{2}$ values were 4.912 and 8.018 , and the $P$ values were 0.027 and 0.005 , respectively.)

Conclusion Dual-energy CT imaging technology can clearly and accurately display urate deposition in patients with gout. It demonstrates good reliability and a high clinical application value.

\section{Introduction}

Gout is a common immune-related disease caused by hyperuricemia due to disorders of purine metabolism or impaired uric acid excretion ${ }^{[1]}$. Precipitation of urate crystals occurs when blood uric acid levels continue to rise above their saturation level in the body. The deposition of urate crystals not only triggers acute attacks of gout, but long-term deposition can lead to the formation of gout stones, which can cause bone destruction, space narrowing, deformity, and dysfunction in the joints ${ }^{[2]}$. In severe cases, gouty nephropathy and renal failure can develop, seriously affecting the quality of life of patients ${ }^{[3]}$. Therefore, the early diagnosis of gout is particularly important. Clinical manifestations, laboratory tests, and imaging examinations are the main methods used to diagnose gout. These commonly used imaging diagnostic techniques have their advantages and disadvantages. With improvements in public health awareness and developments in modern medical technology, noninvasive, convenient, and effective examination methods are gaining more and more attention. Dual-energy CT (DECT) imaging can specifically differentiate urate crystals according to differences in tissue attenuation by emitting rays from two different energy bulbs ${ }^{[4]}$. This study aims to analyze the diagnostic value of DECT imaging in gout urate crystals and provide a basis and guidance for the noninvasive clinical diagnosis of this disease. 


\section{Information And Methods}

\subsection{Subjects}

Data were collected from 83 patients with a suspected diagnosis of gout who were admitted to the Department of Rheumatology at Shanxi Baiqiu'en Hospital between June 2019 and December 2020. There were 74 males and 9 females, aged 22 to 81 years, with a mean age of $40.6 \pm 13.5$ years. Their disease duration ranged from 1 day to 15 years, and the mean disease duration was $4.2 \pm 1.6$ years. Blood uric acid levels ranged from 167.5 to $842 \mu$, with a mean blood uric acid level of $491 \pm 130 \mu \mathrm{mol} / \mathrm{L}$. Patient inclusion criteria for the study were as follows: $₫$ patients who underwent DECT with complete clinical, laboratory, and imaging data; and $\otimes$ patients who were informed about the study and signed the associated consent form with the approval of the medical ethics committee of our university. Patients were excluded from the study in the following cases: $\otimes$ patients with secondary gout due to renal failure, hematological disease, malignant tumors, or lead poisoning; $₫$ patients who were unable to cooperate with clinicians to undertake history taking and physical examination due to serious mental illness; $\mathbb{Z}$

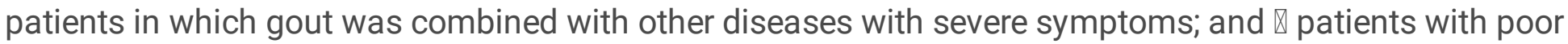
compliance.

\subsection{DECT examination and film reading methods}

DECT of the affected joint area was performed in all patients. A dual-energy CT scanner (SOMATOM Definition Flash from Siemens, Germany) was used for scanning and parameter setting: detailed specific operating parameters need to be added. Based on the three-material decomposition algorithm, DECT images of bone, urate crystals, and soft tissue were simultaneously visualized and given different pseudo-colors. If the green marker appeared in the joint cavity, periarticular synovial capsule, soft tissue, tendon, and ligament, it was assumed to be urate crystals, and the DECT result was considered positive. If the green marker appeared in the areas of motion, vascular calcification, and those areas with a thicker stratum corneum (such as the skin, nails, and heel), it was assumed to be artifact, and the DECT result was considered negative.

Three attending imaging physicians experienced in clinical practice completed the DECT results independently, using a double-blind method. In cases where diagnostic results were inconsistent, conclusions were drawn according to the principle of majority rule.

\subsection{Gold standard examination}

All patients underwent the gold standard diagnostic test: the puncture extraction of joint fluid or gout stone crystals. The diagnosis of gout was confirmed by the discovery of needle-shaped birefringent urate crystals under a polarized light microscope; those without crystals were considered non-gouty.

\subsection{Clinician assessment}


Three attending rheumatologists used history taking and physical examination to assess urate crystal deposition (including the site and amount of involvement) in patients with confirmed gout. The assessments were made without knowledge of the DECT images and according to the principle of majority rule in the case of disagreement.

\subsection{Observation indicators}

The location of the crystals was observed and recorded, and the diagnostic value was assessed by sensitivity, specificity, negative predictive value, positive predictive value, and kappa value. These values were calculated as follows: sensitivity = number of true-positive cases/(number of true-positive cases + number of false-negative cases) $\times 100 \%$; specificity $=$ number of true-negative cases/(number of truenegative cases + number of false-positive cases) $\times 100 \%$; negative predictive value $=$ number of truenegative cases/(number of false-negative cases + number of true-negative cases) $\times 100 \%$; and positive predictive value $=$ number of true-positive cases $/$ (number of true-positive cases + number of true-negative cases) $\times 100 \%$.

\subsection{Statistical treatment}

The collected data were statistically analyzed using SPSS 21.0, and the measurement data that satisfied the normal distribution were expressed as $\overline{\mathrm{x}} \pm \mathrm{s}$. An independent samples $\mathrm{t}$-test was used to compare the two data sets. Count data were expressed as a composition ratio or percentage, and an $\chi^{2}$ test was used for comparison. The ROC curves were constructed, and the area under the curve and the $95 \%$ confidence interval $(\mathrm{Cl})$ were calculated. $\mathrm{P}<0.05$ was considered a statistically significant difference.

\section{Results}

\subsection{Diagnostic value of DECT for urate crystals in gout patients}

Of the 83 patients with suspected gout, 65 cases were diagnosed as gout and 18 as non-gout by intraarticular puncture; gout was diagnosed by DECT imaging in 63 cases and non-gout in 20 cases. The sensitivity of DECT imaging for gout diagnosis was $92.31 \%$, specificity was $83.33 \%$, negative predictive value was $75 \%$, positive predictive value was $95.24 \%$; kappa value was 0.73 ; and the area under the ROC curve was $0.88(95 \% \mathrm{Cl}, 0.77$ to $0.99, P<0.01)$. These results are presented in Table 1 and Fig. 1.

Table 1 Comparison of results of diagnosis of gout by DECT examination and gold standard examination $(n=83$, cases) 


\begin{tabular}{|llll|}
\hline \multirow{2}{*}{ DECT examination } & \multicolumn{2}{c|}{ "Gold standard" examination } & Total \\
\cline { 2 - 3 } & Patients & Non-patients & \\
\hline Positive & 60 (true positive) & 3 (false positive) & 63 \\
\hline Negative & 5 (false negative) & 15 (true negative) & 20 \\
\hline Total & 65 & 18 & 83 \\
\hline
\end{tabular}

2.2 Comparison of DECT with clinical physical examination in the detection of urate crystals

DECT was performed at the self-reported pain site in 65 gout patients, and the percentage of urate crystals deposited in the foot and ankle, knee, wrist, and elbow joints was $98.21 \%(55 / 56), 100 \%(34 / 34)$, $92.00 \%(23 / 25)$ and $83.33 \%(5 / 6)$, respectively. DECT revealed a total of 502 urate crystal deposits, which were irregular in shape, mostly punctate, but also nodular and patchy. The largest deposit measured 19 $\mathrm{mm} * 10 \mathrm{~mm} * 3 \mathrm{~mm}$ and the smallest $2 \mathrm{~mm} * 1 \mathrm{~mm} * 1 \mathrm{~mm}$. The deposits were distributed as follows: 281 $(55.98 \%)$ in the foot and ankle joint, $72(14.34 \%)$ in the knee joints, $134(26.69 \%)$ in the hand and wrist joints, and 15 (2.99\%) in the elbow joints.

The clinicians identified 114 sites of urate crystal deposition based on the history taking and physical examination of patients. This included 67 sites $(58.77 \%)$ in the foot and ankle joints, 8 sites $(7.02 \%)$ in the knee joints, 35 sites $(30.70 \%)$ in the hand and wrist joints, and 4 sites $(3.51 \%)$ in the elbow joints. The number of urate crystals detected by DECT imaging was 4.40 times higher than the number detected by clinical physical examination.

2.3 Comparison of the detection rate of joint lesions by DECT in different stages of gout onset The detection rates of urate crystals and bone destruction by DECT examination were higher in patients with chronic intermittent gout than in patients with acute gout $(P<0.05)$, as shown in Table 2 .

Table 2 DECT examination on the detection rate of joint lesions in patients with gout at different stages [n (\%)]

\begin{tabular}{|llll|}
\hline Onset stage & Number of cases & Urate crystals & Bone destruction \\
\hline Acute phase & 28 & $20(71.4)$ & $9(32.1)$ \\
\hline Chronic intermittent period & 37 & $35(94.6)$ & $25(67.6)$ \\
$\chi^{2}$ & & 4.912 & 8.018 \\
P-value & & 0.027 & 0.005 \\
\hline
\end{tabular}

\section{Discussion}


Gout is one of the most common inflammatory joint diseases ${ }^{[5]}$ and is characterized by the deposition of monosodium urate crystals in synovial fluid and tissues, with disorders of purine metabolism, hyperuricemia, and excessive activation of inflammatory vesicles as its main pathological basis ${ }^{[6]}$. Clinical manifestations include recurrent acute arthritis due to urate crystal irritation; chronic arthritis due to long-term recurrent irritation by urate crystals, gout stone formation, and joint deformity; and gouty nephropathy, in addition to hyperuricemia ${ }^{[7]}$. In recent years, the number of gout patients has increased significantly due to rapid economic development, changes in people's lifestyle and diet, and the utilization of medications, such as diuretics ${ }^{[8]}$. In addition, gout patients are often accompanied by abnormalities in body metabolism and are prone to the combination of hypertension, obesity, hyperlipidemia, type 2 diabetes, and metabolic syndrome, leading to an increased incidence of cardiovascular diseases, such as heart failure, coronary heart disease, pulmonary embolism, deep vein thrombosis, and stroke ${ }^{[9]}$. Patients' quality of life gradually decreases as the disease progresses, and gout is also positively associated with depression ${ }^{[10]}$. Therefore, early diagnosis and timely intervention of gout are particularly important to reduce complications and joint dysfunction ${ }^{[11]}$.

Elevated blood uric acid levels are an important indicator for the diagnosis of gout. However, only 5-12\% of hyperuricemia develops into gout, and about $40 \%$ of people with acute gout do not have elevated blood uric acid levels. This makes it more challenging to diagnose gout based on hyperuricemia alone. The specific manifestation of gout diagnosis is urate crystal deposition, and the previous gold standard for gout diagnosis was synovial fluid aspiration or gout stone microscopy. The 2018 edition of the EULAR guideline also recommends synovial fluid examination and urate crystal microscopy whenever available or when the diagnosis is unclear ${ }^{[12]}$. However, this method is an invasive operation with low patient acceptability and requires a high level of physician skill for puncture and microscopy. In addition, some small joints are difficult to sample, and the technique may be impossible to perform, leading to an increase in false negatives and possible bleeding and infection. X-rays, CT, MRI, and ultrasound are also helpful in the diagnosis of gout. However, $X$-rays can only provide a diagnostic basis for more extensive bone destruction. In the early stages of a gout attack, X-rays only show the swelling of soft tissue around the joints, often with no abnormal relationship between the joints, and they cannot effectively distinguish urate crystals from surrounding tissues. Therefore, $\mathrm{X}$-rays are less sensitive in diagnosing early-stage gout and are only sensitive to nodules with more obvious calcification in middle and late-stage gout ${ }^{[13]}$. Conventional CT can show subtle changes in bone structure and can clearly show gout stones. However, this technique does not show the structures in the bone marrow cavity and the surrounding soft tissue and has some limitations for early gout diagnosis. MRI has the advantage of high tissue resolution and can detect intra-marrow cavity changes, synovitis, cartilage damage, and swelling of the soft tissue around joints in early-stage patients and can image multiple angles, directions, and sequences. However, its specificity is poor, its display rate of calcification is low, it cannot effectively distinguish gouty arthritis from other inflammatory joint diseases, and it is time-consuming and expensive ${ }^{[14]}$. Ultrasound can show tiny urate crystals, synovitis, synovial hyperplasia, joint effusion, and bone destruction and can distinguish urate from pyrophosphate crystals, which is important for the differential diagnosis of gout. 
Ultrasound is radiation-free, inexpensive, and easy to observe dynamically. However, with a more limited field of view, ultrasound is highly dependent on the physician's operating technique. Ultrasound methods also demonstrate somewhat poor reproducibility, inaccurate measurement of gout stone volume, and an inability to accurately assess the relationship between gout stones and surrounding tissues ${ }^{[15]}$.

DECT is a new noninvasive diagnostic imaging technology developed in recent years, which can distinguish between different substances according to their energy attenuation characteristics. Based on the three-material decomposition algorithm, DECT can scan the examined area simultaneously and rapidly using two sets of bulbs with different energy outputs. The two sets of detectors, corresponding to different tissue attenuation characteristics, will acquire two different sets of data, which will be transferred to the supporting workstation for three-dimensional (3D) reconstruction to obtain a set of tissue-specific images with relatively complete information. Different colored markers are used to distinguish between different substances. In general, the bone cortex is marked in blue, the bone cancellous in purple, and the urate crystals in green. This provides a basis for the qualitative diagnosis of the disease and enables physicians to distinguish the urate crystals from the surrounding tissues and accurately assess the site, quantity, and extent of their deposition. In addition, the combination of dualenergy 3D fusion subtraction images and software measurement tools can measure and calculate the volume of urate crystals, identify the smallest crystals less than $3 \mathrm{~mm}$ in diameter ${ }^{[16]}$, and calculate the total amount of urate crystals deposited in a single joint or in the entire scan area. By permitting assessment of the urate crystal load deposited in the joint area, DECT plays a role in quantitative analysis and provides an important basis for early diagnosis and dynamic monitoring of the disease. The above features have led to the necessity and importance of DECT examinations being highlighted in the 2018 EULAR recommendations for gout diagnosis ${ }^{[12]}$.

In this study, among 83 patients with suspected gout, 65 were diagnosed with gout, and 18 were diagnosed with non-gout by synovial fluid aspiration or gout stone microscopy. In comparison, 63 were diagnosed with gout, and 20 were diagnosed with non-gout by DECT imaging. The sensitivity of DECT imaging for the diagnosis of gout was $92.31 \%$, the specificity was $83.33 \%$, the negative predictive value was $75.00 \%$, and the positive predictive value was $95.24 \%$. The results were consistent with those of $\mathrm{Yu}$ et al. ${ }^{[17]}$. They performed DECT and gold standard examinations on 64 patients with suspected gout and confirmed that 58 of them were gout patients, with a sensitivity of $93.1 \%$ and a specificity of $100.0 \%$. The possible reasons for false negatives were the initial onset of the disease, the duration of the disease being less than four weeks, and the effect of uric acid-lowering therapy. The possible reason for false positives was advanced knee osteoarthritis in some patients. In this study, the kappa value was 0.73 , and the area under the ROC curve was $0.88(95 \% \mathrm{Cl}, 0.77-0.99, \mathrm{P}<0.01)$, indicating that DECT imaging technology has good sensitivity, specificity, and accuracy in the diagnosis of gout, and is in strong agreement with the gold standard and has a high diagnostic value. As the DECT examination is noninvasive, rapid, and less operator-dependent, it can replace the gold standard to a certain extent. Therefore, DECT can be used as an important noninvasive diagnostic tool for gout. In this study, DECT detected 502 urate crystals, which was 4.40 times more than the number detected by clinical physical 
examination (114), and was consistent with the findings of Duan ${ }^{[18]}$ and Choi ${ }^{[19]}$. This indicates that DECT has a stronger and more sensitive ability to detect urate crystals and can identify some urate crystal deposits that are difficult to detect earlier by physical examination, providing a basis for timely clinical intervention. The detection rate of urate crystals and bone destruction in patients with chronic intermittent gout was higher than that in the acute stage, indicating that DECT is more effective in detecting urate crystals in patients with chronic intermittent gout. This may be related to the longer disease duration and larger urate crystal deposits in patients with chronic intermittent gout. In future studies, the sample size can be expanded to verify these results further. It was found that the location of gout stone deposition has a sizable impact on the formation and severity of bone destruction, and the more urate crystals deposited in the bone, the more severe the bone destruction. In general, it is more common for bone destruction to be located outside the joint than inside the joint. Intraosseous gout stones have the greatest impact on the formation of bone destruction, followed by intra-articular gout stones. Intra-soft tissue gout stones have the least impact ${ }^{[20]}$. Chhana et al. ${ }^{[21]}$ performed pathological examinations and DECT on two cadavers: an 82-year-old gout patient and an 89-year-old patient without gout. The comparative results showed that the DECT findings were consistent with the pathological findings and that there was a high correlation between bone destruction and the location of the gout stones.

The shortcomings of this study are as follows: (1) the sample size was insufficient, and there may be errors between the results and the overall; (2) the follow-up time was short, and the results of urate crystal volume changes were not collected and analyzed; and (3) there was no comparison with other imaging techniques. In the follow-up study, we should strengthen multi-center cooperation, increase the sample size, use multiple methods for comparison or joint examination, and follow up the investigation study for a more extended period.

\section{Conclusion}

In summary, this study confirms that DECT imaging technology is a reliable technique that demonstrates high sensitivity and specificity in the diagnosis of gout. Results are comparable to those achieved by gold standard tests, and therefore, it can be concluded that DECT is effective in reducing misdiagnosis and missed diagnosis. The examination takes less time, is less expensive, and is less operator-dependent than other methods, and as DECT is noninvasive, it is readily accepted by patients. DECT imaging technology can accurately detect the site, quantity, and volume of urate crystals in the patient's body, which is beneficial to the physician's assessment of bone destruction. DECT helps assess the patient's condition dynamically, providing a basis for follow-up treatment, which has a high clinical application value.

\section{Abbreviations}

DECT:Dual-energy CT 
Cl:confidence interval

3D:three-dimensional

\section{Declarations}

\section{Ethics approval and consent to participate}

This study was conducted in accordance with the Declaration of Helsinki,Oral consent for patient participation was obtained and approved by the ethics committee of Fenyang College of Shanxi Medical University .

\section{Consent for publication}

Not applicable.

\section{Availability of data and materials}

All data generated or analyzed during this study are included in this published article.

\section{Competing interests}

All of the authors had no any personal, financial, commercial, or academic conflicts of interest separately.

\section{Funding}

Innovation and Entrepreneurship Training Program for College Students of Shanxi Province (2018817);

Social Development Field Project of Luliang Municipal Science and Technology Bureau (2020SHFZ44)

\section{Authors' contributions}

LYconceived of the study, and XL and HL participated in its design and coordination and LQ helped to draft the manuscript. All authors read and approved the final manuscript.

\section{Acknowledgements}

Not applicable

\section{References}

1. Chu YL, Jiang YQ, Sun SL, et al. The differential profiles of long non-coding RNAs between rheumatoid arthritis and gouty arthritis[J]. Discovery Medicine, 2017, 24(132): 133-146. 
2. Bang D, Xu J, Keenan R, et al. Cardiovascular disease prevalence in patients with osteoarthritis, gout or both[J]. Bull Hosp Jt Dis,2016;74(2):113-8.

3. Li Xueyuan, Jiang Meihua, Li Wei, et al. Research progress of early kidney injury diagnostic biomarkers in gout patients[J]. Shandong Medicine, 2019, 59(14): 99-102.

4. Wei L, Lu ZW, Liao Y, et al. Diagnostic value of dual-energy CT in gouty arthritis [J]. Chinese Journal of Medical Physics, 2019,36(05): 568-570.

5. Edward R, Hyon K. C. Epidemiology of Gout[J]. Clin Rheum Dis, 2014, 40(2): 155-175.

6. Pascart T, Liote F. Gout: state of the art after a decade of developments[J]. Rheumatology, 2019, 58(1): 27-44.

7. Wang N, Zhang JW, Wang T, et al. Clinical characteristics of acute gouty arthritis combined with type 2 diabetes mellitus[J]. Journal of Xi'an Jiaotong University (Medical Edition), 2020, 41(03): 390-395.

8. Sun MY, Ana IV, Richard JR, et al. Untangling the complex relationships between incident gout risk, serum urate, and its comorbidities[J]. Arthritis Research \& Therapy, 2018, 20(1): 90.

9. Multidisciplinary Consensus Expert Group on the Treatment of Hyperuricemia-Related Diseases. Multidisciplinary expert consensus on the diagnosis and treatment of hyperuricemia-related diseases in China[J]. Chinese Journal of Internal Medicine, 2017, 56(3): 235-248.

10. Lin S, Zhang HQ, Ma AG. Association of gout and depression: a systematic review and Metaanalysis[J]. Int J Geriatr Psychiatry, 2018, 33(3): 441-448.

11. Guo Yefeng. Diagnostic value of dual-energy CT processing technique in the deposition of urate crystals in patients with gouty arthritis[J]. Medical Clinical Research, 2018, 35 (04): 763-765.

12. Richette P, Doherty M, Pascual E, et al. 2018 updated European League Against Rheumatism evidence-based recommendations for the diagnosis of gout[J]. Ann Rheum Dis, 2019, 79(1): 31-38.

13. Kupfer S, Winklhofer S, Becker AS, et al. Gouty arthritis: Can we avoid unnecessary dual-energy CT examinations using prior radiographs?[J]. PLoS One, 2018, 13(7): e0200473.

14. Lennart J, Isabelle DK, Nele H, et al. Dual-energy CT: a new imaging modality for bone marrow oedema in rheumatoid arthritis[J]. Ann Rheum Dis, 2018, 77(6):958-960.

15. Gamala M, Linn-rasker SP, Nix M, et al. Gouty arthritis: decision-making following dual-energy CT scan in clinical practice, a retrospective analysis[J]. Clin Rheumatol, 2018, 37(7):1879-1884.

16. Wang HY, Liu ZH. New advances in the comprehensive diagnosis and treatment of gout[J]. Chinese Journal of Metallurgical Industry Medicine, 2020, 37(05): 511-513.

17. Yu X. H., Du L. X., Chen C. P., et al. The value of dual-energy CT imaging in the early diagnosis of gouty arthritis[J]. Journal of Medical Imaging. 2019, 29(1): 124-126

18. Duan Yanfeng, Li Wei, Yang Wei, et al. Analysis of the value of dual-source CT dual-energy imaging for the diagnosis of gout nodule deposition[J]. Chongqing Medicine, 2017, 46(04): 491-493.

19. Choi HK, Al-arfaj AM, Eftekhari A, et al. Dual energy computed tomography in tophaceous gout[J]. Ann Rheum Dis, 2009, 68(10): 1609-1612. 
20. Tekaya R, Tekaya AB, Saidane 0 , et al. Tophaceous hip gouty arthritis revealing asymptomatic axial gout [J]. Egypt Rheumatologist, 2018, 40 (03): 209-212.

21. Chhana A, Doyle A, Sevao A, et al. Advanced imaging assessment of gout: comparison of dualenergy CT and MRI with anatomical pathology[J]. Ann Rheum Dis, 2018, 77(4): 629-630.

\section{Figures}

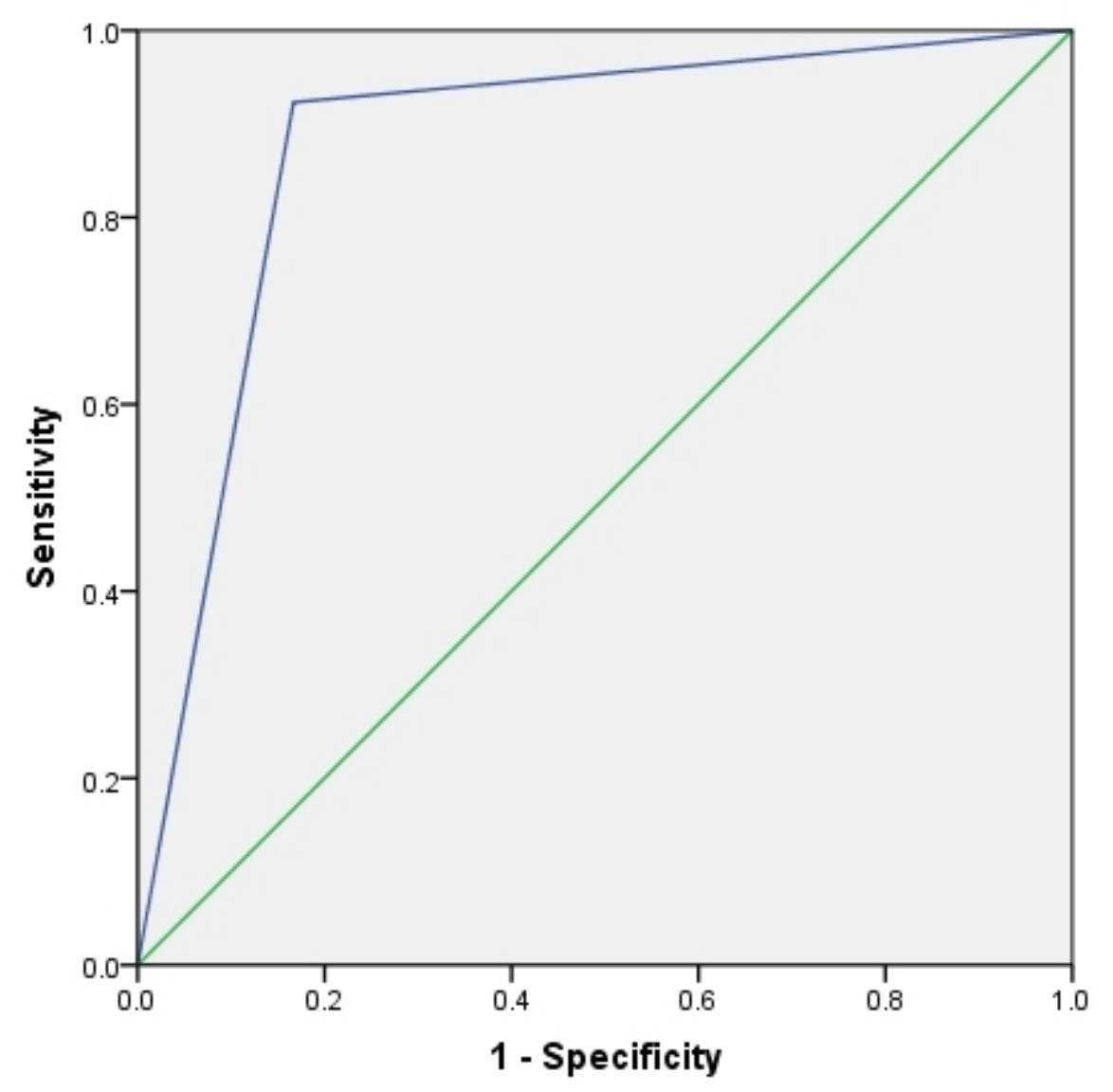

\section{Figure 1}

ROC curve of dual-energy CT imaging technique for diagnosing gout 